\title{
Comparación diagnóstica entre mastografía digital y
} tomosíntesis mamaria

\section{Diagnostic comparison between digital mastography and breast}

\section{tomosynthesis}

Sandra M. Matehuala-Montúfar* y Carolina González-Vergara

Departamento de Radiología e Imagen, Hospital Ángeles Mocel, Ciudad de México, México

\section{RESUMEN}

Introducción: El tamizaje del cáncer de mama con mamografía convencional tiene limitaciones conocidas. La tomosíntesis es una técnica en la que se adquieren imágenes a bajas dosis de radiación y desde múltiples ángulos. La información obtenida reduce la superposición de estructuras, permitiendo una mejor evaluación, detección, caracterización y localización de las lesiones. Objetivo: Comparar la proporción diagnóstica entre mastografía digital y tomosíntesis. Método: Estudio observacional, retrospectivo, analítico y de corte transversal. Se evaluaron 430 expedientes electrónicos con imágenes de mastografía digital y tomosíntesis, se efectuó análisis de doble lectura y se comparó la visibilidad de hallazgos por ambos métodos de imagen de acuerdo con BI-RADS. Se realizó una diferencia de proporciones en la que se consideró como significativo un valor de $\mathrm{p}<0.05$. Resultados: Se encontró una diferencia estadísticamente significativa al comparar los hallazgos al analizar únicamente las imágenes obtenidas por mastografía digital en 137 casos, frente a los 400 casos con hallazgos visibles documentados en imágenes de tomosíntesis en una segunda lectura. Se obtuvo un valor $Z$ de 1.8, con $p=0.0329$. Conclusiones: Se halló un valor de proporción diagnóstica de la tomosíntesis mayor que el obtenido con la mastografía digital convencional.

Palabras clave: Tomosíntesis mamaria. Mastografía digital. Mastografía de diagnóstico. 


\section{ABSTRACT}

Introduction: Screening for breast cancer with conventional mammography has known limitations. Tomosynthesis is a technique were images are acquired with low radiation doses from multiple angles. The obtained information reduces the superposition of structures, allowing for a better evaluation, characterization, and localization of injuries. Objective: To compare the proportional diagnosis between digital mammography and tomosynthesis. Method: Observational study, retrospective, with transversal cut. 430 electronic records were evaluated with images from digital mammography and tomosynthesis, a double reading analysis was made, comparing the visibility of findings by both methods according to BI-RADS. A difference in proportions was made where a significance level is $p<0.05$. Results: A statistically significance difference was found when comparing the images from digital mammography in 137 cases versus the 400 cases with visible findings documented in images from tomosynthesis upon a second reading. A value for $Z=1.8$ was obtained, with a value for $p=0.0329$. Conclusions: A higher proportional value was obtained from tomosynthesis than from conventional digital mammography.

Keywords: Breast tomosynthesis. Digital mammography. Diagnostic mammography.

\section{INTRODUCCIÓN}

Se ha demostrado que el tamizaje mamario organizado y realizado de manera óptima y oportuna tiene el potencial de reducir entre un $20 \%$ y un $40 \%$ la tasa de mortalidad por cáncer de mama, siendo la mastografía el método de detección que ha demostrado un mayor impacto en esta cuando se efectúa con calidad $^{1-4}$.

La mastografía digital (mamografía 2D) en el tamizaje permite mejorar la sensibilidad en la detección temprana del cáncer de mama; sin embargo, sus limitaciones son bien conocidas, provenientes en su mayoría de la superposición de estructuras mamarias normales, así como de la mama densa, ya que es una imagen obtenida de manera bidimensional, lo que dificulta su interpretación por la presencia de imágenes compuestas 5,6 . La sensibilidad de la mastografía oscila entre el $71 \%$ y el $91 \%$ en mamas con patrón graso, mientras que su especificidad se encuentra entre el 55\% y el 98\%, disminuyendo proporcionalmente al aumentar la densidad del tejido mamario ${ }^{1}$.

La tomosíntesis, también conocida como mamografía 3D, es una técnica en la que se adquieren imágenes a bajas dosis de radiación y desde múltiples ángulos a medida que el tubo de rayos $X$ se mueve a través de un arco con movimiento que varía entre $15^{\circ}$ y $60^{\circ}$, dependiendo del fabricante. Los datos de estas imágenes son reconstruidos en cortes de $1 \mathrm{~mm}$, en la orientación de la adquisición, siendo el número de cortes proporcional al espesor de la mama comprimida ${ }^{4-8}$. 


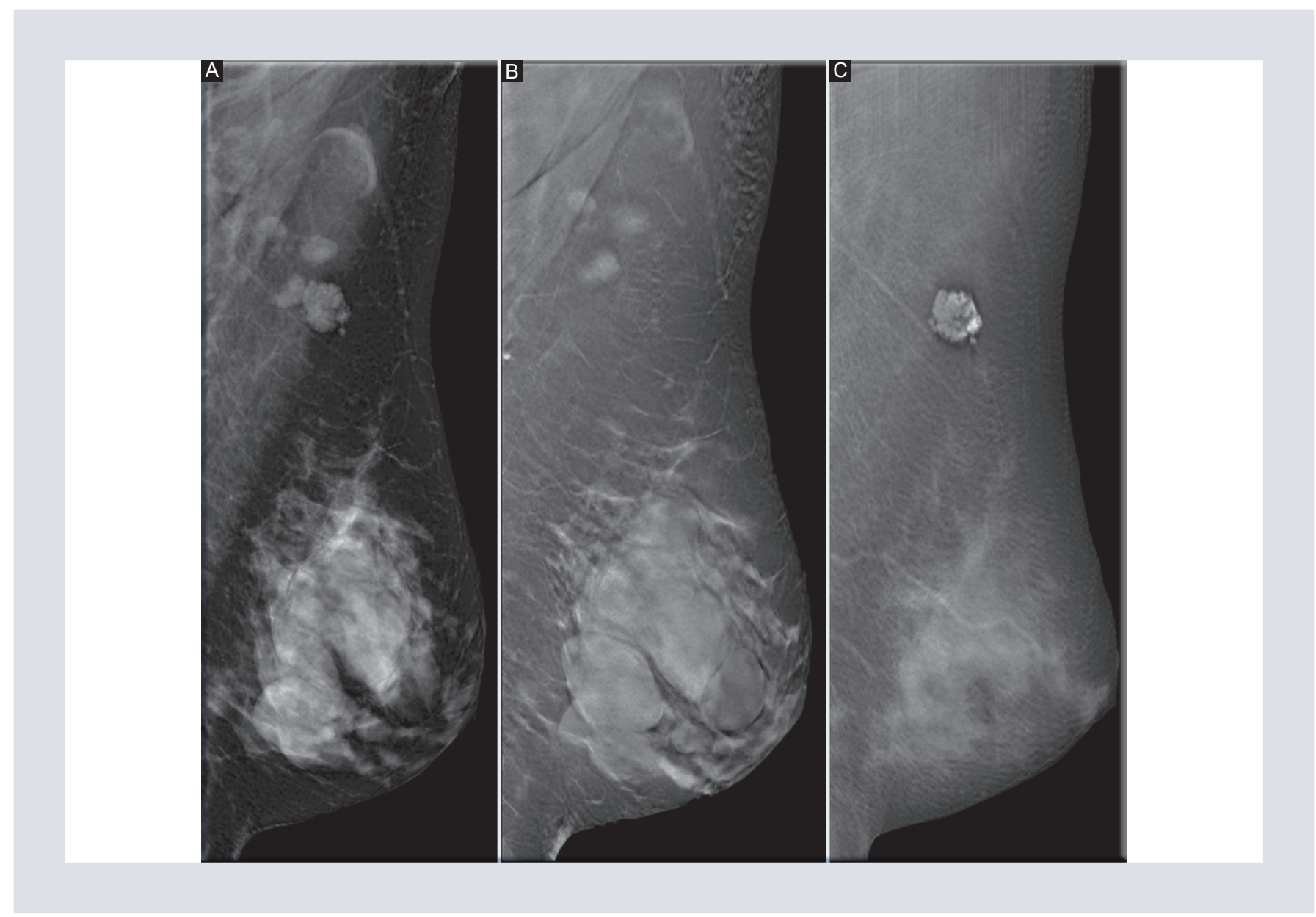

Figura 1. Proyecciones medio-lateral-oblicuas de la mama izquierda con patrón mamario extremadamente denso. A: imagen de mastografía convencional en la que se observan nódulos de bordes oscurecidos, así como un nódulo microlobulado proyectado en axila. B y C: imágenes subsecuentes de tomosíntesis, en las que se documentan múltiples nódulos circunscritos con densidad intermedia. El nódulo axilar, visible en los cortes más superficiales de la piel, muestra un halo claro hipodenso de aire, en relación con un nevo verrugoso; ambos de aspecto benigno.

La información obtenida de la adquisición de la tomosíntesis reduce la superposición de estructuras, permitiendo una mejor evaluación, detección, caracterización y localización de las lesiones ${ }^{7-9}$ (Fig. 1), y disminuyendo de manera importante las tasas de rellamado ${ }^{4,5,8,10,11}$. Como beneficio adicional, reduce la necesidad de obtener mayor número de proyecciones complementarias o estudios adicionales, lo que la hace un estudio más rentable en la práctica clínica en relación con la mastografía digital $^{12-15}$. Ha demostrado una mejor especificidad y una mayor sensibilidad mamográfica debido a la mejor visibilidad de las lesiones, principalmente en mujeres con alta densidad mamaria ${ }^{6-9,16-19}$ (Fig. 2), incrementando la tasa de detección oportuna de cáncer de mama ${ }^{4,10,12,13}$.

El objetivo de este estudio es comparar la proporción diagnóstica entre la mastografía digital y la tomosíntesis en la caracterización de las lesiones mamarias según la clasificación BI-RADS (Breast Imaging Reporting and Data System), quinta edición. 


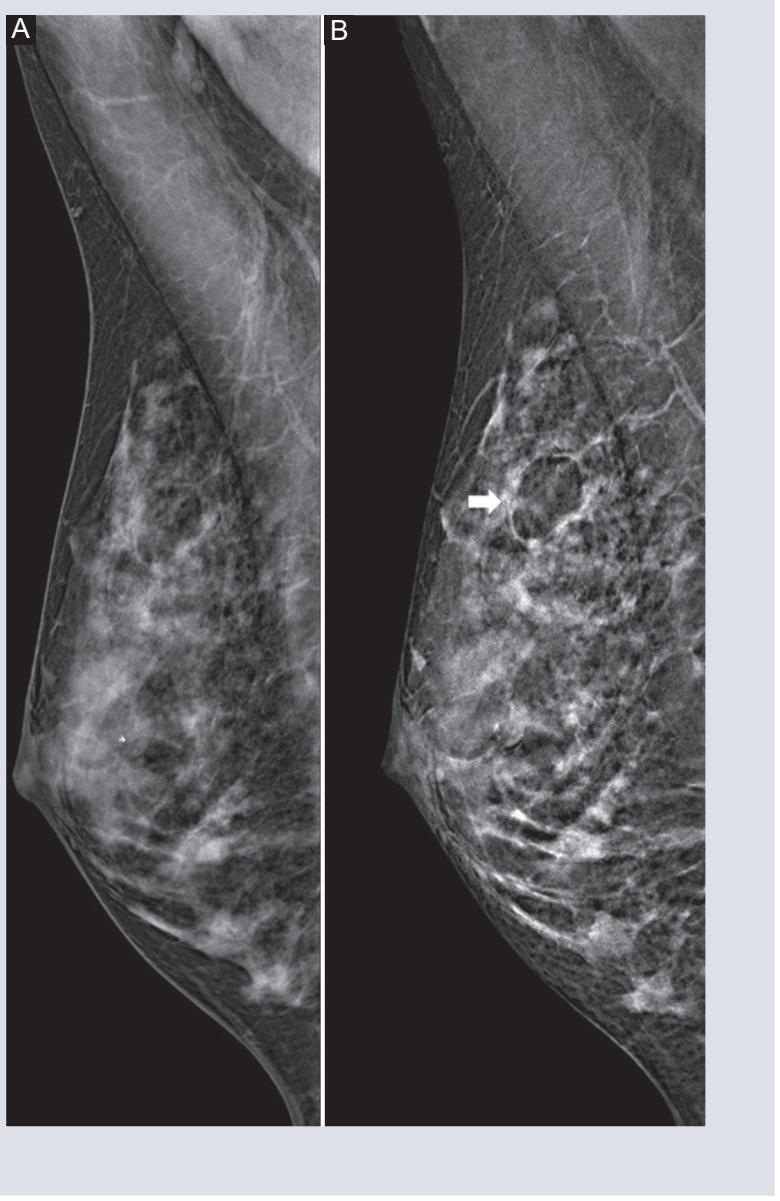

Figura 2. Proyecciones medio-lateral-oblicuas de la mama derecha. A: imagen de mastografía convencional en la que se observa la mama con parénquima fibroglandular tipo $C$ del ACR. B: en la imagen de tomosíntesis queda delimitado un nódulo ovalado, circunscrito, hipodenso respecto al parénquima glandular, localizado en el tercio medio de los cuadrantes superiores, sugestivo de un lipoma (flecha).

\section{MÉTODO}

Se realizó un estudio observacional, retrospectivo, analítico y de corte transversal, recolectando información de 439 mujeres que acudieron al Hospital Ángeles Mocel para realización de mastografía y tomosíntesis, entre enero de 2019 y enero del 2020.

Se incluyeron mujeres mayores de 36 años que contaban con mastografia digital obtenida en tres proyecciones: céfalo-caudal (CC), céfalocaudal extrema (XCC) y medio-lateral-oblicua (MLO); así como con tomosíntesis en dos proyecciones, CC y MLO, de manera bilateral. Se excluyeron las pacientes con antecedente personal de mastectomía o mastoplastia con implantes.

Los estudios de mastografía (2D y 3D) se realizaron con un equipo GIOTTO CLASS serie 30000, modelo de doble columna, el cual cuenta con un ánodo en el tubo de rayos $X$ constituido por una aleación de berilio, níquel, tungsteno y renio, con un filtro de plata.

Los valores de $\mathrm{kV}$ y $\mathrm{mAs}$ se definieron automáticamente en función al grosor de la mama comprimida sobre el detector automático.

Se realizó una doble adquisición con dosis efectivas totales dentro de los rangos permitidos de acuerdo con la NOM 029 SSA 2002, por debajo de los $0.3 \mathrm{mSv}$. Las imágenes de mastografía digital se obtuvieron colocando el tubo de rayos $X$ perpendicular a la mama en un solo disparo de baja dosis de radiación. Las imágenes de tomosíntesis se adquirieron con una angulación del tubo de rayos $X$ de $30^{\circ}$ sobre la mama, realizando un disparo de baja dosis de radiación por cada grado de angulación en 11 ocasiones, para la posterior reconstrucción automática de imágenes definitivas consecutivas de $1 \mathrm{~mm}$ de espesor; el número de imágenes reconstruidas dependió del grosor de la mama al comprimirla.

La evaluación se realizó por dos radiólogos expertos en imagen mamaria, con 5 y 30 años de experiencia y conocimiento en el cribado mamario. 

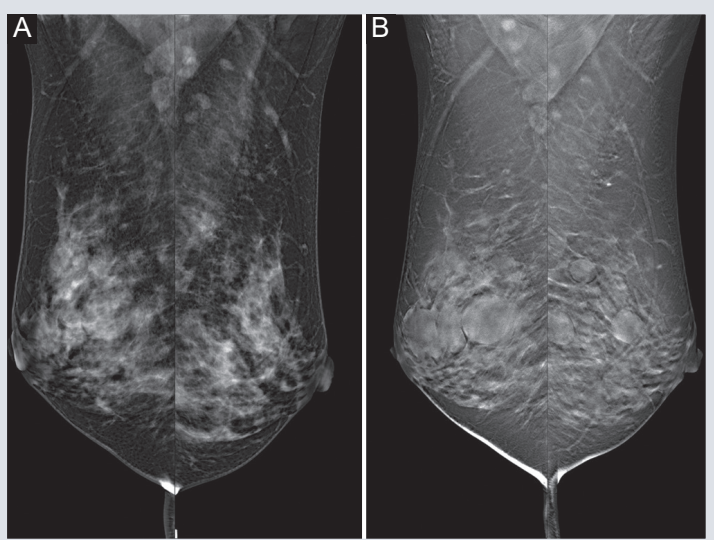

Figura 3. Proyecciones medio-lateral-oblicuas bilaterales. A: mamografía que muestra unas mamas heterogéneamente densas, con nódulos bilaterales de bordes oscurecidos.

B: tomosíntesis en la que se reduce la superposición de tejido normal y se delimitan los bordes de los nódulos, circunscritos y de características benignas.

Las imágenes se analizaron en la misma estación de trabajo, que cuenta con dos monitores de alta resolución (5 $\mathrm{Mpx}$ ) de grado médico, marca Eizo, en un software visualizador específico (Raffaello Mamo 3.6.5) para mastografía y tomosíntesis.

Los casos fueron aleatorizados en varias sesiones de lectura para ambos radiólogos. Se realizó un análisis de doble lectura; en la primera visualización se examinaron las imágenes de mastografía digital convencional para finalmente comparar los hallazgos observados con los encontrados en una segunda lectura que incluía la revisión de imágenes de la mastografía convencional y las imágenes de tomosíntesis. Ambas radiólogas estuvieron de acuerdo en los diagnósticos finales, sin conocer una el resultado de la otra. Se clasificó un grupo como hallazgos visibles y se incluyeron todas aquellas mastografías (2D o 3D) que permitieron documentar

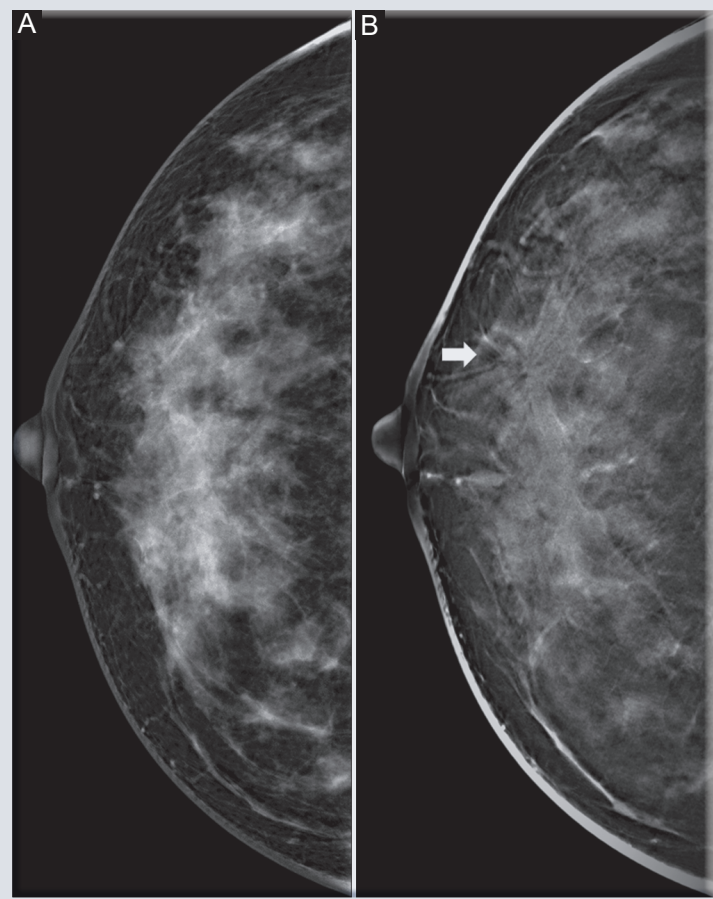

Figura 4. Proyecciones céfalo-caudales de la mama derecha de una paciente sin antecedentes quirúrgicos. A: imagen $2 \mathrm{D}$ en la que se observa un parénquima fibroglandular heterogéneamente denso. B: imagen de tomosíntesis en la que se visualiza una lesión estelar con distorsión de la arquitectura mamaria de los cuadrantes externos, plano superficial (flecha), no observada en la imagen de mastografía convencional. Se recomienda biopsia para análisis histopatológico.

lesiones según las características especificadas en BI-RADS quinta edición (nódulos, distorsiones de la arquitectura, asimetrías y calcificaciones). Se clasificaron como hallazgos no visibles las imágenes mastográficas en las que no era posible identificar lesiones (Figs 3-4). La densidad mamaria también fue calificada de acuerdo con los cuatro patrones mamarios descritos por el American College of Radiology (ACR).

La información recabada se consignó en una base de datos en Excel, incluyendo la fecha de adquisición del estudio, la edad de la 
paciente, el patrón mamográfico, el BI-RADS otorgado, los hallazgos mastográficos y su visibilidad en las modalidades 2D y 3D. Se exportó al software estadístico SPSS versión 12.

En la estadística descriptiva, las variables se describieron dependiendo de su naturaleza: las cualitativas (hallazgos visibles, hallazgos no visibles, BI-RADS y patrón mamario) en términos de frecuencias absolutas (número de casos) y frecuencias relativas (porcentajes), y las cuantitativas (edad) con medidas de tendencia central.

La caracterización de las lesiones en las imágenes obtenidas por mastografía digital y tomosíntesis se evaluó con estadística inferencial. A las variables cualitativas nominales de los dos grupos se les realizó una prueba de hipótesis para dos proporciones. Para evaluar la asociación se realizó una prueba de $\chi^{2}$, tomándose como resultado estadísticamente significativo un valor de $\mathrm{p}<0.05$.

Los autores declaramos que no existen conflictos éticos, debido a que la información fue obtenida del archivo digital.

\section{RESULTADOS}

Se incluyeron 430 expedientes radiológicos digitales que contenían imágenes de mastografía digital y de tomosíntesis que cumplían con los criterios de inclusión. En cuanto a la comparación entre los hallazgos observados en la primera y la segunda lecturas, se presentó la siguiente distribución: 138 hallazgos visibles por mastografía digital convencional, 293 sin hallazgos visibles por mastografía digital convencional, 400 hallazgos visibles con complemento de tomosíntesis y 30 hallazgos no visibles por este último método de imagen. Con estos datos se realizó un análisis estadístico para diferencia de proporciones, en el que se obtuvo un valor $Z$ de 1.8, que corresponde a un valor $\mathrm{p}=0.0329$, lo cual resulta estadísticamente significativo.

Las pacientes incluidas tuvieron una media de edad de 52 años (rango: 36-86 años). El BI-RADS 2 fue el mayormente encontrado, en 290 casos (67.4\%), y el patrón mastográfico más frecuente fue el tipo C-ACR, en 182 (42.3\%) (Tabla 1). Se encontró correlación entre los patrones mamarios densos (C-D del ACR) y el número de hallazgos observados por mastografía 3D. En 182 pacientes con patrón mamario tipo $\mathrm{C}$ del ACR se documentaron 142 (78\%) casos con hallazgos por tomosíntesis que no eran visibles en las imágenes de mastografía digital convencional, mientras que en 74 pacientes con patrón mamario tipo D del ACR la utilidad de la tomosínstesis se demostró en 57 (77\%) casos (Fig. 5).

\section{DISCUSIÓN}

El cáncer de mama es catalogado como uno de los principales problemas de salud pública, tanto localmente como en todo el mundo, motivo por el cual la Organización Mundial de la Salud tiene como objetivo implementar normas que impacten en su incidencia, teniendo en cuenta los recursos económicos limitados de muchas naciones. Por ello, los métodos empleados deben ser efectivos en el diagnóstico de esta enfermedad para evitar el desgaste de los recursos. México cuenta con un programa 
TABLA 1. Características clínicas y mastográficas en 430 mujeres evaluadas con mastografía digital convencional (2D) y tomosíntesis (3D).

\begin{tabular}{l|c|c|}
\hline $\begin{array}{l}\text { Edad (años) } \\
\text { Mínima } \\
\text { Media }\end{array}$ & 36 & DE: 35.35 \\
Máxima & 61 & \\
\hline BI-RADS & 86 \\
0 & \\
1 & $36(8.4 \%)$ & \\
2 & $0(0 \%)$ & \\
3 & $290(67.4 \%)$ & \\
4 & $76(17.7 \%)$ & $p^{*}$ \\
5 & $22(5.1 \%)$ & 1.000 \\
6 & $4(0.9 \%)$ & 0.046 \\
\hline Patrón mamario (ACR) & $2(0.5 \%)$ & 0.737 \\
A & & \\
B & $23(5.3 \%)$ & 0.784 \\
C & $151(35.1 \%)$ & \\
D & $182(42.3 \%)$ & \\
\hline DE: desviación estándar. & $74(17.2 \%)$ & \\
*Correlación con la prueba de $\chi^{2}$, del patrón mamario y la imagen mastográfica \\
(2D y 3D). \\
Los valores se presentan como media (mínimo-máximo) o como frecuencia \\
absoluta (\%).
\end{tabular}

de tamizaje con el objetivo de identificar de manera temprana el cáncer de mama. Este es controvertido, ya que no contempla otras modalidades imagenológicas de manera habitual. Si la tomosíntesis se agregara como complemento a la mastografía, permitiría una reducción en la tasa de falsos positivos y de procedimientos innecesarios ${ }^{3,6,7}$.

En nuestro estudio se demostró que la edad de las pacientes (media de 52 años) y el patrón más frecuentemente encontrado (tipo C del ACR) se relacionan con lo reportado por Phi Xa et al. ${ }^{17}$ quienes indican que la tasa de detección del cáncer de mama es baja solo por mastografía, por lo que realizar el tamizaje adicionando tomosíntesis disminuye el número de casos de rellamado e incrementa la detección de lesiones ${ }^{17}$. La utilidad de la tomosíntesis para la

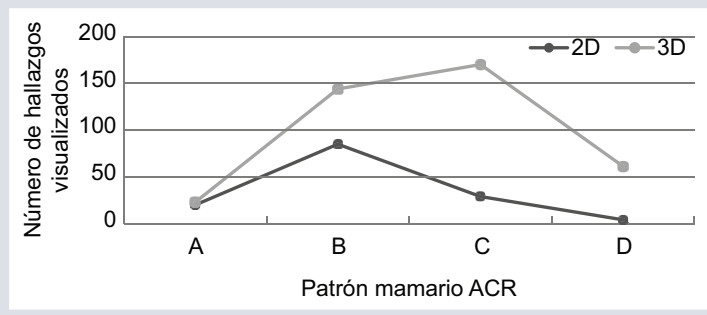

Figura 5. Correlación entre el patrón mamario mastográfico y el número de hallazgos visualizados por mastografía digital convencional (2D) y tomosíntesis (3D) en 430 mujeres.

visualización de lesiones mastográficas fue valorada cualitativamente basándose en una doble lectura ${ }^{7}$, en relación con lo realizado en nuestro estudio, en el que se contó con dos radiólogas expertas en patología mamaria, evidenciándose un incremento en la visualización de lesiones no valoradas en la mastografía convencional (Figs. 6 and 7).

En contraste con el estudio realizado por Mancilla y González ${ }^{20}$, en el que evaluaron los patrones mamarios de las mujeres mexicanas documentando una mayor frecuencia del patrón fibroglandular en su población de estudio, nuestros resultados muestran un mayor número de pacientes con patrones mamarios densos. Esta discrepancia podría tener sustento tanto en el tamaño de la muestra como en los criterios de selección.

De los 30 casos mencionados que no fueron beneficiados con el complemento de tomosíntesis, se pudo reconocer que en su mayoría eran pacientes con patrón mamario extremadamente denso, como ha sido demostrado en la literatura por diversos autores, de acuerdo a estudios de Vourtsis A et al y Phi Xa et al. Las lesiones que con mayor frecuencia no 

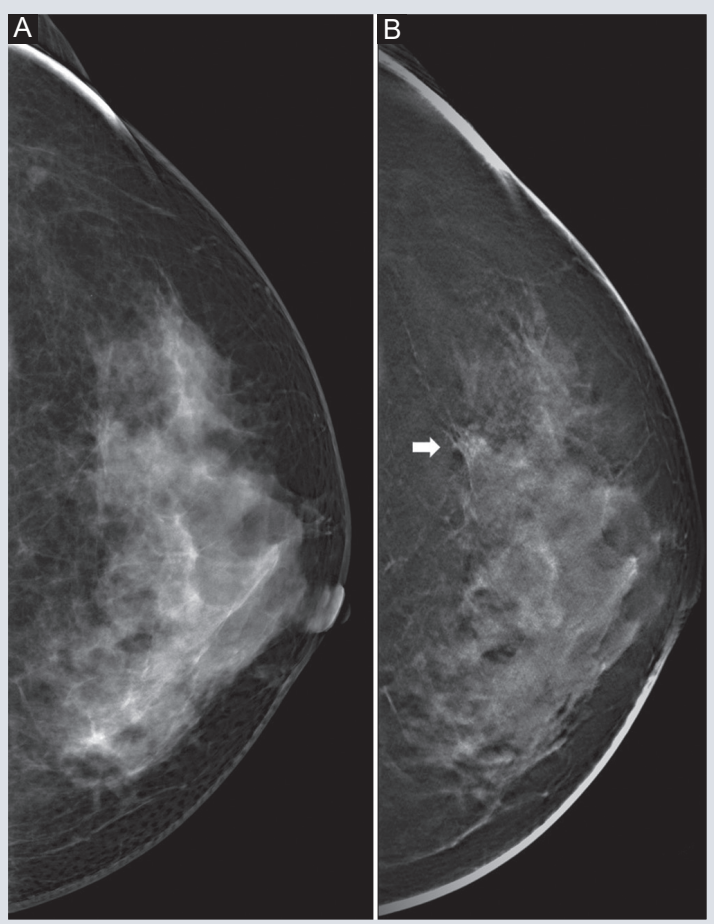

Figura 6. Proyecciones céfalo-caudales de la mama izquierda. A: mastografía $2 \mathrm{D}$ en la que se observa un parénquima fibroglandular heterogéneamente denso. B: imagen de tomosíntesis en la que se visualiza una lesión estelar con distorsión de la arquitectura mamaria asociada a microcalcificaciones de baja densidad de los cuadrantes externos, tercio profundo (flecha). No se observó en la imagen de mastografía convencional. Se clasifica como lesión BI-RADS 4 y se recomienda biopsia para análisis histopatológico.

pudieron visualizarse en este grupo de pacientes fueron los nódulos.

La nula discrepancia en los diagnósticos finales se debió a la homogenización de la información mastográfica basada en el BIRADS, así como a la técnica de doble lectura, como se dicta en la NOM 041 SSA 2011.

Es así como la combinación de mastografía y tomosíntesis muestra un beneficio a nivel particular institucional, siendo lo contrario a nivel poblacional como consecuencia del
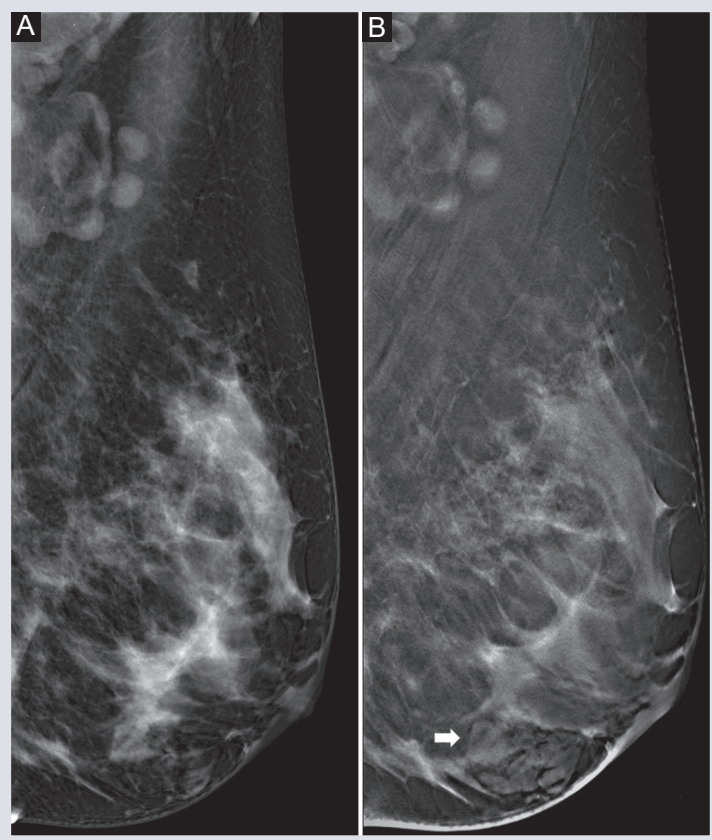

Figura 7. Proyecciones medio-lateral-oblicuas de la mama izquierda. A: imagen de mastografía convencional en la que se observa un parénquima fibroglandular tipo $C$ del ACR. B: en la imagen de tomosíntesis se aprecia un nódulo circunscrito de contornos lobulados con densidad mixta, localizado en el tercio medio de los cuadrantes inferiores (flecha).

pequeño tamaño de las muestras en los estudios publicados $2,5,8,9$.

\section{CONCLUSIONES}

Se obtuvo un valor de proporción diagnóstica de la tomosíntesis mayor que el de la mastografía digital convencional, permitiendo unas mejores visualización y caracterización de las lesiones en las imágenes obtenidas por mastografía, y aportando una mayor utilidad de este método de imagen en las pacientes con patrones mamarios de alta densidad.

Nuestro grupo de trabajo sugiere la realización sistemática de tomosíntesis en los patrones mamarios $\mathrm{C}$ y D del ACR. 


\section{FINANCIAMIENTO}

El presente estudio no requirió financiamiento.

\section{CONFLICTO DE INTERESES}

Los autores declaran que no existe conflicto de intereses.

\section{RESPONSABILIDADES ÉTICAS}

Protección de personas y animales. Los autores declaran que para esta investigación no se han realizado experimentos en seres humanos ni en animales.

Confidencialidad de los datos. Los autores declaran que han seguido los protocolos de su centro de trabajo sobre la publicación de datos de pacientes.

\section{Derecho a la privacidad y consentimiento} informado. Los autores han obtenido el consentimiento informado de los pacientes y/o sujetos referidos en el artículo. Este documento obra en poder del autor de correspondencia.

\section{BIBLIOGRAFÍA}

1. Prevención, tamizaje y referencia oportuna de casos sospechosos de cáncer de mama en el primer nivel de atención. Guía de evidencias y recomendaciones: guía de práctica clínica. México: CENETEC; 2017. Disponible en: http: / / cenetec-difusion.com/gpc-sns / ?p=490
2. Norma Oficial Mexicana NOM-041-SSA2-2011, para la prevención, diagnóstico, tratamiento, control y vigilancia epidemiológica del cáncer de mama. Disponible en: http://www.cenetec.salud.gob.mx/descargas/equipoMedico/normas/NOM_041_SSA2_2011.pdf

3. Tabár L, Yen AMF, Wu WYY, Chen SLS, Chiu SYH, Fann JCY, et al. Insights from the breast cancer screening trials: how screening affects the natural history of breast cancer and implications for evaluating service screening programs. Breast J. 2015;21:13-20.

4. Niell BL, Freer PE, Weinfurtner RJ, Arleo EK, Drukteinis JS. Screening for breast cancer. Radiol Clin North Am. 2017;55:1145-62.

5. Vourtsis A, Berg WA. Breast density implications and supplemental screening. Eur Radiol. 2019;29:1762-77.

6. Friedewald SM. Breast tomosynthesis: practical considerations. Radiol Clin North Am. 2017;55:493-502.

7. Mall S, Lewis S, Brennan P, Noakes J, Mello-Thoms C. The role of digital breast tomosynthesis in the breast assessment clinic: a review. J Med Radiat Sci. 2017;64:203-11

8. Chong A, Weinstein SP, McDonald ES, Conant EF. Digital breast tomosynthesis: concepts and clinical practice. Radiology. 2019;292:1-14.

9. Hooley RJ, Durand MA, Philpotts LE. Advances in digital breast tomosynthesis. Am J Roentgenol. 2017;208:256-66.

10. Rocha García AM, Mera Fernández D. Breast tomosynthesis: state of the art. Radiologia. 2019;61:274-85.

11. Sujlana PS, Mahesh M, Vedantham S, Harvey SC, Mullen LA, Woods RW. Digital breast tomosynthesis: image acquisition principles and artifacts. Clin Imaging. 2019;55:188-95.

12. Peppard HR, Nicholson BE, Rochman CM, Merchant JK, Mayo RC, Harvey JA. Digital breast tomosynthesis in the diagnostic setting: indications and clinical applications. Radiographics. 2015;35:975-90.

13. Fiorica JV. Breast cancer screening, mammography, and other modalities Clin Obstet Gynecol. 2016;59:688-709.

14. Lee CI, Cevik M, Alagoz O, Sprague BL, Tosteson ANA, Miglioretti DL, et al. Comparative effectiveness of combined digital mammography and tomosynthesis screening for women with dense breasts. Radiology. 2015;274:772-80.

15. Kalra VB, Wu X, Haas BM, Forman HP, Philpotts LE. Cost-effectiveness of tomosynthesis in annual screening mammography. Am J Roentgenol. 2016;207:1152-5.

16. González CSI, Gracia QJF, Gascón MA, Lavín AR, Hernández BLD, Barrera LFA. Detección en tomosíntesis de distorsiones en la arquitectura ocultas en mamografía. Rev Mex Mastol. 2019;9:10-2.

17. Phi XA, Tagliafico A, Houssami N, Greuter MJW, de Bock GH. Digital breast tomosynthesis for breast cancer screening and diagnosis in women with dense breasts - a systematic review and meta-analysis. BMC Cancer. 2018;18:1-9.

18. Conant EF, Barlow WE, Herschorn SD, Weaver DL, Beaber EF, Tosteson ANA, et al. Association of digital breast tomosynthesis vs digital mammography with cancer detection and recall rates by age and breast density. JAMA Oncol. 2019;5:635-42.

19. Poplack S. Breast tomosynthesis: clinical evidence. Radiol Clin North Am. 2017;55:475-92.

20. Mancilla-Mazariegos ST, González-Vergara C. Patrones mastográficos en las mujeres mexicanas. Rev An Radiol Mex. 2019;17:1-3. 